\title{
Ambiente Colaborativo para Avaliação de Cadeias de Abastecimento
}

\author{
Paula Prata ${ }^{1}$, Paulo Fazendeiro ${ }^{1}$, Carlos Augusto ${ }^{1}$, Susana Azevedo 2 ,
} V. Cruz-Machado 3

\{pprata, pandre\}@di.ubi.pt, \{a23669, sazevedo\}@ubi.pt, vcm@fct.unl.pt

${ }^{1}$ Instituto de Telecomunicações (IT), Dep. de Informática, Universidade da Beira Interior 6200-001 Covilhã, Portugal

${ }^{2}$ UNIDEMI - Dep. de Economia e Gestão, Universidade da Beira Interior

6200-209 Covilhã, Portugal

3 UNIDEMI- Dep. de Engenharia Mecânica e Industrial, Faculdade de Ciências e Tecnologia da

Universidade Nova de Lisboa, 2829-516 Caparica, Portugal

DOI: 10.4304/risti.12.1-15

Resumo: Neste trabalho é proposto um ambiente colaborativo que permite a medição do desempenho de uma cadeia de abastecimento (bem como o dos seus elos constituintes) no atual contexto empresarial caracterizado pela competitividade, turbulência e globalização dos mercados. Os índices propostos são genéricos e a sua adaptação a diferentes cadeias de abastecimento é imediata. As tecnologias da cloud são analisadas segundo a perspetiva das mais-valias que as podem promover enquanto suporte para o modelo de avaliação proposto. São ponderados os requisitos do modelo organizacional colaborativo proposto, resultando num modelo de dados flexível também apresentado. A utilidade do conceito é demonstrada com o desenvolvimento de um protótipo de aplicação na infraestrutura de cloud EC2 da Amazon.

Palavras-chave: Gestão de Cadeias de Abastecimento; Cloud Computing; Ambientes Colaborativos; Integração de Sistemas.

\section{Collaborative Environment for Supply Chain Assessment}

Abstract: This paper proposes a collaborative environment that allows the performance measurement of a supply chain (as well as its constituent links) in the current business environment, which is characterized by competitiveness, turbulence and globalization of the markets. The proposed indices are generic and their adaptation to different supply chains is straightforward. The cloud technologies are analyzed from the perspective of the assets that can promote them as a support for the proposed evaluation model. The analysis of the requirements of the proposed collaborative organizational model, results in a flexible data model (also presented). The usefulness of the concept is 
demonstrated with the development of a software prototype in the Amazon EC2 cloud infrastructure.

Keywords: Supply Chain Management; Cloud Computing; Collaborative Environments; Systems Integration.

\section{Introdução}

Uma cadeia de abastecimento, supply chain em inglês (SC), pode ser entendida como uma organização (de empresas) altamente colaborativa com uma dinâmica de rede reconfigurável. Esta rede de empresas que liga vários agentes, desde o fornecedor até ao consumidor final passando pela fabricação e serviços, tem por objetivo gerir de forma eficaz os fluxos físicos, financeiros e informacionais para assim atingir os objetivos do negócio (Stevens, 1989). A competitividade de um dado produto ou serviço não é resultado de uma única unidade organizacional mas sim o efeito da coordenação e otimização das atividades ao longo de toda a cadeia de abastecimento a que essa unidade pertence. Isto é, a competição deixou de se verificar apenas entre empresas individuais e centra-se agora nas próprias cadeias de abastecimento.

Surge assim a gestão da cadeia de abastecimento que consiste na coordenação estratégica dos vários elementos que a compõem com o objetivo de melhorar o desempenho de cada empresa individual e da SC como um todo (Mentzer et al., 2001). É de esperar que uma SC bem gerida consiga fornecer ao cliente o produto certo, com as especificações exigidas, no sítio certo e dentro do prazo definido, ao menor custo possível para todos os membros da SC (Lambert, Cooper \& Pagh, 1998).

Novos paradigmas de gestão têm surgido com o objetivo de melhorar a competitividade das SCs através do aumento da qualidade dos produtos e da melhoria do serviço oferecido aos clientes (Espadinha-Cruz et al., 2011). Neste trabalho vai ser dado especial destaque aos paradigmas Lean, Ágil, Resiliente e Verde. Paralelamente a gestão de conhecimento tem sido reconhecida como crucial para o crescimento e desenvolvimento das organizações (Xavier et al., 2012). Em particular a capacidade para integrar, construir e reconfigurar competências internas e externas é essencial para lidar com ambientes em rápida mudança. A avaliação do nível de satisfação dos diversos paradigmas é um requisito comum a cada um destes processos e como tal é também essencial à gestão eficiente das SCs e consequentes ganhos de produtividade. Existem já trabalhos sobre os quatro paradigmas aqui abordados (Azevedo, Carvalho, \& Cruz-Machado, 2012) mas apresentam uma abordagem mais de gestão com implicações a nível da medição de desempenho da cadeia, não explorando, quer o ambiente colaborativo, quer as potencialidades da cloud.

O principal objetivo deste trabalho é propor um modelo colaborativo para acomodar de forma integrada todo o processo de avaliação da SC. A cloud é aqui explorada como meio de operacionalizar e suportar o modelo proposto. Um contributo adicional advém da generalização da metodologia para medir o comportamento ágil de uma SC (Azevedo et al., 2012) aos outros paradigmas e consequente eliciação de um índice compósito para cada paradigma considerado.

Na secção 2 deste artigo é brevemente revista uma seleção de paradigmas de gestão das SCs. Na secção 3 são enunciados os aspetos que promovem as tecnologias de cloud 
computing como uma solução emergente de apoio a este modelo organizacional. A secção 4 propõe um modelo de avaliação de comportamentos de uma SC. Na secção 5 é feita a análise dos requisitos do sistema de informação que permite concretizar o modelo colaborativo de avaliação descrito e na secção 6 apresentamos o protótipo desenvolvido. Finalmente, a secção 7 apresenta a conclusão e propostas para trabalho futuro.

\section{Paradigmas de gestão das cadeias de abastecimento}

Segundo Christopher (2005), a gestão das cadeias de abastecimento é a gestão das relações a montante e a jusante com os fornecedores e clientes criando valor ao cliente ao menor custo. Para Mentzer (2001) a cadeia de abastecimento é simplesmente um conjunto de empresas, tanto a montante (ou seja, a oferta), como a jusante (ou seja, a distribuição) incluindo o consumidor final. Num ambiente competitivo em constante mutação, em que o ciclo de vida dos produtos é cada vez mais curto, novos paradigmas de gestão das cadeias de abastecimento têm surgido. Dentre estes destacamos pela sua pertinência os paradigmas lean, ágil, resiliente e verde. Estes paradigmas têm despertado o interesse da comunidade académica, quer em termos individuais (Cumbo, Kline \& Bumgardner , 2006; EPA, 2000; Holt \& Ghobadian, 2009; Naylor, Naim, \& Berry, 1999; Zhu, Sarkis, \& Geng, 2005), quer no seu conjunto pelo facto de se apresentarem determinantes para o desempenho das empresas e respetivas cadeias de abastecimento (Azevedo, Carvalho, \& Cruz-Machado, 2012).

Uma SC lean é aquela que tenta desenhar os seus produtos de forma a minimizar os desperdícios de recursos e maximizar o lucro. A abordagem de gestão lean, desenvolvida por Taiichi Ohn na Toyota Motor Corporation, no Japão, é a base para o Sistema de Produção Toyota (TPS), assente em dois pilares principais: "Autonomação ou Jidoka" e "Just-in-Time" (JIT) de produção (Ohno, 1998). De acordo com Womack \& Jones (1991) a estratégia lean é uma abordagem que defende a utilização de menos recursos (menos esforço humano, menos equipamento, menos tempo e menos espaço) na produção de produtos que estejam mais próximos das necessidades dos clientes. Há evidências de que a tendência de muitas empresas é procurar soluções de baixo custo, por causa da pressão sobre as margens, podendo levar a serem mais lean, porém tornam-se cadeias de abastecimento mais vulneráveis (Azevedo et al, 2008; Peck, 2005; Christopher \& Peck, 2004).

Uma SC ágil é aquela que é capaz de responder rapidamente a alterações não previsíveis dos mercados, quer em termos de quantidade, quer de variedade (Agarwal, Shankar \& Tiwari, 2007; Christopher, 2008). Segundo (Angulo \& Martín, 2009) a agilidade é um requisito indispensável para garantir a sobrevivência das empresas e o sucesso das organizações num ambiente tão turbulento, competitivo e globalizado como o atual.

Uma SC resiliente é aquela que é capaz de reagir a alterações ou roturas abruptas e inesperadas. Em termos de gestão de SCs, a resiliência é definida como a capacidade de a SC ser capaz de se regenerar para o seu estado original ou para um novo estado mais favorável após um distúrbio (Christopher \& Peck, 2004). O objetivo da cadeia de abastecimento é de entregar o produto certo, na quantidade certa, na condição certa, no lugar certo, na hora certa, pelo custo certo. Uma vez que as necessidades dos 
clientes estão em constante mudança, as cadeias de abastecimento devem estar adaptáveis às mudanças futuras e responder adequadamente às exigências do mercado.

Finalmente, uma SC verde é uma SC que incorpora preocupações ambientais nas suas práticas de gestão visando a minimização dos impactos no ambiente (Rao \& Holt, 2005). Ambientalmente sustentável a gestão da cadeia de abastecimento verde surgiu como filosofia organizacional para alcançar fins e objetivos corporativos na quota de mercado, reduzindo riscos e impactos ambientais, melhorando a eficiência ecológica destas empresas e dos seus parceiros (Zhu, Sarkis \& Lai, 2008; Rao \& Holt, 2005). Shuwang (2005) considera que as cadeias de abastecimento verdes são um requisito do desenvolvimento sustentável e uma forma eficaz para as empresas enfrentarem os desafios da concorrência no mercado. De acordo com Srivastava (2007), uma gestão verde de SCs pode reduzir o impacto ecológico da atividade industrial sem sacrificar a qualidade, o custo, a fiabilidade, o desempenho ou a eficiência energética.

\section{Cloud e Gestão de Cadeias de Abastecimento}

Tendo em conta que atualmente os contextos económico e empresariais se alteram muito rapidamente, o modelo de cloud computing aparece como a tecnologia adequada para permitir a partilha de informação entre todos os parceiros de uma SC. A computação em cloud consiste num novo modelo de negócio onde os consumidores podem ter acesso a hardware e software, através da internet, em que apenas pagam os recursos de que necessitam em cada instante e apenas quando os utilizam (Armbrust et al., 2010).

De acordo com a definição do National Institute of Standards and Technology (Mell \& Grance, 2011) a computação em cloud tem 5 caraterísticas principais: (i) on demand self-service, o utilizador pode configurar e aceder a capacidades de computação sem necessidade de interação humana com o fornecedor do serviço; (ii) broad network access, os recursos estão disponíveis na rede através de mecanismos standard, como os protocolos da internet, que promovem o uso de plataformas cliente heterogéneas (laptops, smartphones, tablets, etc.); (iii) resource pooling, os recursos de computação são agrupados para servir vários consumidores através de um modelo multi-tenant com diferentes recursos físicos e virtuais atribuídos. Exemplos de recursos são armazenamento, processamento, memória e largura de banda de rede; (iv) rapid elasticity, as capacidades de computação podem ser alocadas ou libertadas elasticamente, em alguns casos de forma automática, para escalonar mais ou menos recursos de acordo com a maior ou menor procura; (v) measured service, os sistemas de cloud monitorizam, controlam e reportam automaticamente o uso dos recursos.

De acordo com Kefer (2012) através da cloud as companhias de uma SC poderão colaborar a uma escala global, com menos riscos e menos custos do que com sistemas de software tradicionais. Pequenas empresas não terão de fazer grandes investimentos em infraestruturas e recursos humanos de tecnologias da informação (TI). São identificadas três formas de a cloud melhorar as operações de uma SC: (i) uma aplicação na cloud pode oferecer visibilidade de toda a SC em tempo real; (ii) transferir as aplicações para a cloud, implica criar normas para a representação da informação entre todos os elementos da SC e definir regras de segurança comuns; (iii) uma plataforma de cloud permite construir uma comunidade colaborativa. 
Sendo o sistema proposto neste trabalho fornecido na cloud segundo o modelo SaaS Software como um Serviço (Armbrust et al., 2010), as empresas apenas terão de alugar um serviço de software, sem se preocuparem nem com a sua manutenção técnica nem com problemas de escala do serviço. Além da redução de custos de desenvolvimento e manutenção, é importante notar que a arquitetura multi-tenant da cloud permite também que diferentes SCs adiram à plataforma, partilhando funcionalidades ao mesmo tempo que detêm o controlo sobre os seus dados e sobre os seus membros. Através da plataforma proposta cada empresa pode obter uma visão global da sua SC, e todos os elementos da SC poderão colaborar para melhorar o desempenho e competitividade do seu negócio. As atuais tecnologias Web fornecem os meios para construir a plataforma enquanto o modelo de negócio da cloud fornece o modo de distribuir e reduzir os custos.

\section{Avaliação do Comportamento de Cadeias de Abastecimento}

A avaliação de cada comportamento lean, ágil, resiliente e verde (ou outros que venham a ser propostos) é baseada na determinação de um conjunto de práticas relevantes para o comportamento em estudo. Por exemplo, uma prática para o comportamento verde será qualquer ação executada ao longo da cadeia que tenha como efeito eliminar ou reduzir algum tipo de impacto negativo no meio ambiente. Essas práticas poderão ser identificadas a partir da literatura, ou sugeridas por um conjunto de peritos na área do comportamento em estudo.

Esses peritos serão associados à SC e além de serem os responsáveis pela identificação das práticas que determinam um certo comportamento, serão também responsáveis pela atribuição de pesos relativos a cada uma dessas práticas. Essa atribuição de pesos relativos a cada prática poderá ser feita, por exemplo, seguindo o método Delphi (Linstone \& Turoff, 1975). Neste método cada perito classifica por ordem de importância as várias práticas e após cada ronda de inquéritos os resultados são compilados e tidos em consideração para a ronda seguinte. Após um número de rondas pré-definido é calculado o peso relativo de cada prática.

Generalizando o índice proposto em (Azevedo et al., 2012) podemos quantificar o comportamento $X$ de uma empresa se, para cada prática associada a esse comportamento, for possível aferir o nível de implementação da empresa. Esse nível de implementação será medido usando uma escala de Likert (Likert, 1932) de 5 níveis, em que o valor 1 significa "prática não implementada" e 5 significa "prática totalmente implementada”.

O índice do comportamento $X$ para uma empresa, $B X$, representa uma medida do grau de implementação nessa empresa, das várias práticas identificadas como refletindo o comportamento $X$, isto é, como implementando a estratégia $X$. Associando o nível de implementação de cada prática à sua importância relativa dada pelo peso da prática previamente determinado o índice do comportamento $X$ para uma dada empresa será dado pela equação 4.1. 


$$
B X=\frac{\sum_{i=1}^{n} W_{x i} \cdot P_{x i}}{\sum_{i=1}^{n} W_{x i}}
$$

Nesta equação, $P_{x i}$ representa o nível de implementação da prática $i$ para $o$ comportamento $X, W_{x i}$ representa o peso da prática $i$ para o comportamento $X$ e $n$ é o número total de práticas. Finalmente, o índice compósito do comportamento $X$ para uma dada SC $\left(X_{S C}\right)$ é construído a partir dos índices das empresas que formam a SC. Considera-se que numa dada SC, empresas diferentes podem ter importâncias relativas diferentes. Representando por $W_{C j}$ o peso relativo da empresa $j$ dentro da $S C$ considerada, o índice do comportamento $X$ numa dada SC é dado pela equação 4.2,

$$
X_{S C}=\frac{\sum_{j=1}^{m} W_{C j} \cdot B X_{j}}{\sum_{j=1}^{m} W_{C j}}
$$

onde $m$ é o número de empresas que integram a $\mathrm{SC}, B X_{j}$ é o índice do comportamento $X$ para a empresa $j$ e $W_{C j}$ é o peso relativo da empresa $j$ na cadeia considerada. Uma vez que o índice de um comportamento $X$ para uma empresa é definido por um valor entre 1 e 5, então o valor para o índice de um comportamento para uma SC será também um valor entre 1 e 5 . Um índice de valor 1 significa que a cadeia não implementa quaisquer práticas do comportamento em estudo, e um índice de valor 5 significa que a cadeia implementa completamente todas as práticas. É de notar que, neste modelo, estamos a assumir que o peso de uma empresa numa SC é independente do comportamento que estamos a avaliar e que assumimos que os pesos das práticas para um dado comportamento são comuns a todas as empresas da SC.

\section{Requisitos do Modelo Colaborativo}

Nesta secção é apresentada a análise do sistema de informação necessário para implementar uma plataforma de avaliação do comportamento de SCs. Após a identificação dos tipos de utilizadores e quais os seus âmbitos de atuação, são descritas as funcionalidades do sistema e é proposto um modelo conceptual de dados que permite implementar essas funcionalidades.

As boas práticas no planeamento da arquitetura de informação empresarial (Serra et al., 2008) foram tidas em devida conta garantindo quer o alinhamento das entidades informacionais com os processos de negócio (através da identificação e organização estruturada da informação de interesse para o modelo), quer o alinhamento das aplicações com as entidades informacionais (evitando a criação de zonas de informação duplicada).

O sistema terá quatro tipos de utilizadores. O administrador do sistema, o administrador de uma SC, a empresa e o perito. Um utilizador do tipo "administrador do sistema" é responsável por toda a plataforma e tem acesso total a toda a informação (pode ler, escrever, modificar e apagar). Um utilizador do tipo "administrador de SC" é responsável por uma dada $\mathrm{SC}$ e tem acesso total a toda a informação de todas as empresas que fazem parte dessa SC. Um utilizador do tipo "empresa" é responsável pela informação de uma dada empresa. Tem acesso total à informação da sua empresa 
e pode consultar a informação da SC a que essa empresa pertence. Um utilizador do tipo "perito" é responsável pela identificação das práticas que determinam um certo comportamento e pela atribuição dos seus pesos relativos. Está associado a um determinado tipo de comportamento e tem acesso total às práticas desse comportamento.

\subsection{Funcionalidades}

Para avaliar um determinado comportamento de uma SC, isto é, para calcular o índice de um comportamento, são necessários dois passos prévios: 1 - identificar as práticas relevantes para esse índice; 2 - atribuir os pesos relativos a cada uma das práticas. $\mathrm{Na}$ fase atual da construção da plataforma foi assumido que as práticas são obtidas da literatura sendo o administrador do sistema o responsável pela sua introdução. $\mathrm{O}$ administrador da SC identifica quais as práticas que, para a sua SC, implementam um dado índice e associa cada uma delas à SC e ao índice. Por omissão, o peso da prática será $1 / t$ (onde $t$ representa o número total de práticas desse índice para essa SC). Tendo em conta esta opção, nesta fase do trabalho, as funcionalidades dos utilizadores do tipo "perito" não serão consideradas. É de notar que a extensão do sistema para passar a responder aos passos 1 e 2 referidos acima não implica modificações no modelo de dados apresentado na secção 5.2.

O “administrador do sistema” será responsável pela gestão das seguintes entidades: utilizadores, empresas, SCs, práticas e índices. Para todas elas poderá inserir, modificar, apagar e consultar ocorrências. Será este administrador quem valida o registo dos utilizadores. Quando um utilizador do tipo "empresa" se regista, introduz qual a SC a que pertence. No entanto a relação de pertença da empresa a uma SC só será efetivada quando posteriormente o administrador da SC a validar.

O “administrador da SC" será responsável pelas operações que dizem respeito à sua SC: validar se uma empresa pertence à $\mathrm{SC}$; associar ou retirar índices à $\mathrm{SC}$, associar práticas a um índice da SC, calcular o valor dos índices da SC. Pode ainda aceder a todos os dados das companhias da SC.

O utilizador “empresa” é responsável pelas operações que dizem respeito à empresa: atualizar dados da entidade empresa; inserir nível de implementação das práticas dos índices adotados; calcular os valores dos índices da empresa. O utilizador do tipo "empresa" pode ainda consultar os dados das empresas da SC a que pertence.

\subsection{Modelo Conceptual de Dados}

O modelo conceptual de dados foi construído partindo de cinco tipos de entidades principais representadas a cheio no diagrama da figura 1: empresa (company), utilizador (user), cadeia de abastecimento (SC), índice (index) e prática (practice).

Entre as entidades empresa e utilizador existe uma associação que dará origem à entidade company_user com o significado seguinte: uma empresa tem um ou vários utilizadores, um utilizador corresponde a zero ou uma empresa. Um utilizador não está associado a uma empresa no caso de ser administrador, administrador de uma SC ou perito. 
Entre as entidades empresa e SC existe uma associação com atributos que origina a entidade company_sc. Uma SC possui várias empresas e uma empresa pode pertencer a várias SCs. Por exemplo uma empresa têxtil que produz tecidos para assentos de automóveis, pode pertencer simultaneamente a uma SC têxtil e a uma SC automóvel. Cada ocorrência da entidade company_sc conterá, além dos identificadores da empresa e da SC, o peso relativo (weight_company) e a posição da empresa nessa SC (sc_position). A posição da empresa na SC representa se a empresa é uma empresa focal, um fornecedor de $1^{\mathrm{O}}$ nível, um fornecedor de $2^{\mathrm{o}}$ nível, um distribuidor, etc.

Entre as entidades SC e utilizador existe uma associação que é representada colocando o identificador da SC na tabela da entidade utilizador como chave estrangeira. Uma SC tem vários utilizadores e um utilizador pertence a uma única SC. Os utilizadores do tipo empresa serão associados a uma SC que será uma de entre as várias a que a sua empresa está associada. Note-se que um utilizador do tipo empresa está associado a uma empresa. Na entidade empresa estão os dados da empresa a que pertence e na entidade utilizador estão os seus dados do utilizador. Isto significa que se uma empresa estiver associada a duas ou mais SCs, terá de ter pelo menos um utilizador por cada SC a que esteja associada. Se um utilizador é um perito, a sua $\mathrm{SC}$ será a que corresponde à sua área de conhecimento. Se um utilizador é um administrador de SC, a sua SC será aquela que administra. Se um utilizador é um administrador do sistema então está associado a uma SC global que virtualmente representa o acesso a todas as SCs.

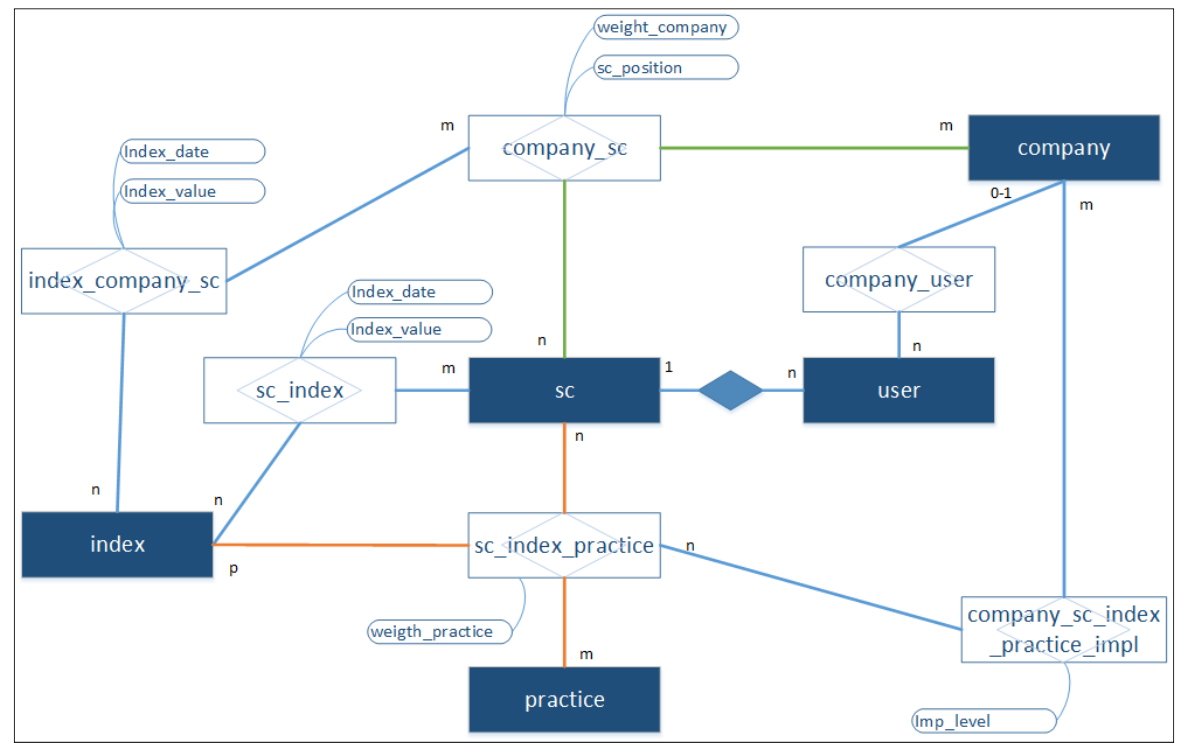

Figura 1 - Modelo de dados

Entre a entidade SC e a entidade índice existe uma associação com atributos que dá origem à entidade sc_index. Para uma SC podem ser calculados vários índices e um índice pode estar associado a várias SCs. A entidade sc_index além dos identificadores das entidades SC e índice vai possuir os atributos index_value e index_date que 
representam o valor calculado para um dado índice de uma SC e a data em que o índice foi calculado.

Entre as entidades índice e company_sc existe uma associação com atributos que representa o valor do índice para uma dada empresa numa dada SC e que é designada por index_company_sc. Um índice pode estar associado a várias ocorrências de company_sc e uma ocorrência da entidade company_sc pode estar associada a vários índices. Cada ocorrência da entidade index_company_sc conterá, além dos identificadores das entidades índice e company_sc, os atributos index_value e index_date que representam o valor calculado para um dado índice de uma empresa quando associada a uma dada SC e a data em que foi calculado.

Entre as entidades SC, índice e prática existe uma associação ternária com atributos que dá origem à entidade sc_index_practice e que representa para uma dada SC e um dado índice quais as práticas que são relevantes. Essa associação, além de conter os identificadores das três entidades que interrelaciona, contém também o atributo weight_practice que representa o valor do peso relativo de cada prática para aquele índice, naquela SC.

Entre as entidades empresa e sc_index_practice vai existir uma associação com atributos que dá origem à entidade company_sc_index_practice_impl e que representa a implementação feita por uma empresa de uma dada prática para uma dada SC e um dado índice. O atributo imp_level representa o valor da implementação da prática.

\section{A Aplicação BAC4SC}

Nesta secção é apresentado o protótipo desenvolvido para estudo do comportamento do modelo colaborativo. A aplicação é designada por (BAC4SC - Behaviour Assessment on the Cloud for Supply Chain) e encontra-se publicada na cloud em http://54.229.2.15/. No protótipo é possível selecionar as práticas relevantes para um dado índice, atribuir o nível de implementação das práticas numa dada empresa e calcular os índices das empresas e das SCs.

Em fase de implementação está a funcionalidade de identificação pelos peritos das práticas relevantes para um dado comportamento, assim como a atribuição do peso relativo de cada prática de uma forma colaborativa pelos representantes das várias empresas de uma dada SC.

De seguida são descritas as tecnologias usadas, a arquitetura da aplicação e apresentadas algumas das páginas web para ilustrar a interface construída. E descrita a infraestrutura de cloud usada para publicar a aplicação e são explicadas as opções de segurança implementadas.

\subsection{Implementação do Protótipo}

O protótipo consiste numa aplicação web em que a interface do lado do cliente foi desenvolvida em XHTML 1.0 e CSS 2.1. A componente da lógica de acesso aos dados do lado do servidor foi desenvolvida em PHP 5.4.7 usando a extensão PHP Data Objects 
(PDO) ${ }^{1}$. A extensão PDO fornece uma camada de abstração standard para interagir com qualquer sistema de base de dados. A base de dados foi implementada em MySQL 5.5.27, o servidor web usado foi o Apache 2.4.3 e a aplicação foi publicada na infraestrutura de cloud Amazon Elastic Compute Cloud (EC2) podendo ser acedida por um qualquer Browser. A EC2 foi escolhida pelas condições para desenvolvimento freeware que permite. Na cloud foi usada uma instância do sistema operativo (SO) Ubuntu server 12.04.2.LTS de 64 bits e criado um grupo de segurança que funcionando como uma firewall permite controlar o tráfego de acesso à instância do SO. Em particular o grupo de segurança criado permite o acesso aos portos 22 para SSH, 80 para HTTP e 443 para HTTPS. Os componentes da aplicação são esquematizados na figura 2. As páginas da aplicação foram desenhadas com o objetivo de cada tipo de utilizador poder ter a sua interface personalizada. De forma dinâmica cada SC terá componentes próprios quer em termos de imagem quer de conteúdo, assim como cada empresa. Na figura 3 apresenta-se a página principal da aplicação onde um utilizador poderá fazer o seu registo e após validação pelo administrador do sistema poderá fazer o login.

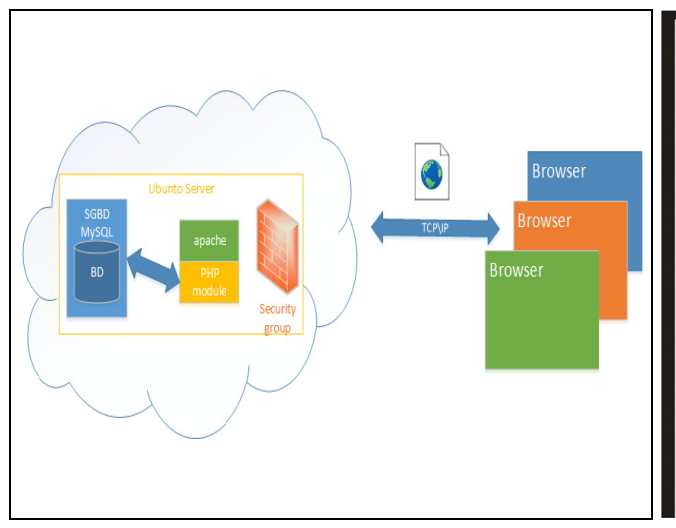

Figura 2 - Componentes da Aplicação

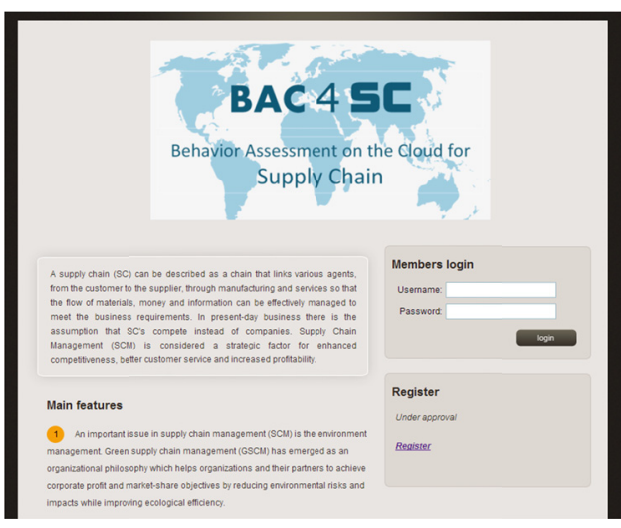

Figura 3 - Página principal

Na figura 4 é mostrada uma das páginas da área de administração da SC automóvel onde são selecionadas as práticas relevantes para o comportamento verde. Na figura 5 é apresentada a página que permite à empresa CompAutoX reportar os seus níveis de implementação das práticas verdes.

${ }^{1}$ PHP Data Objects, http://php.net/manual/en/book.pdo.php 


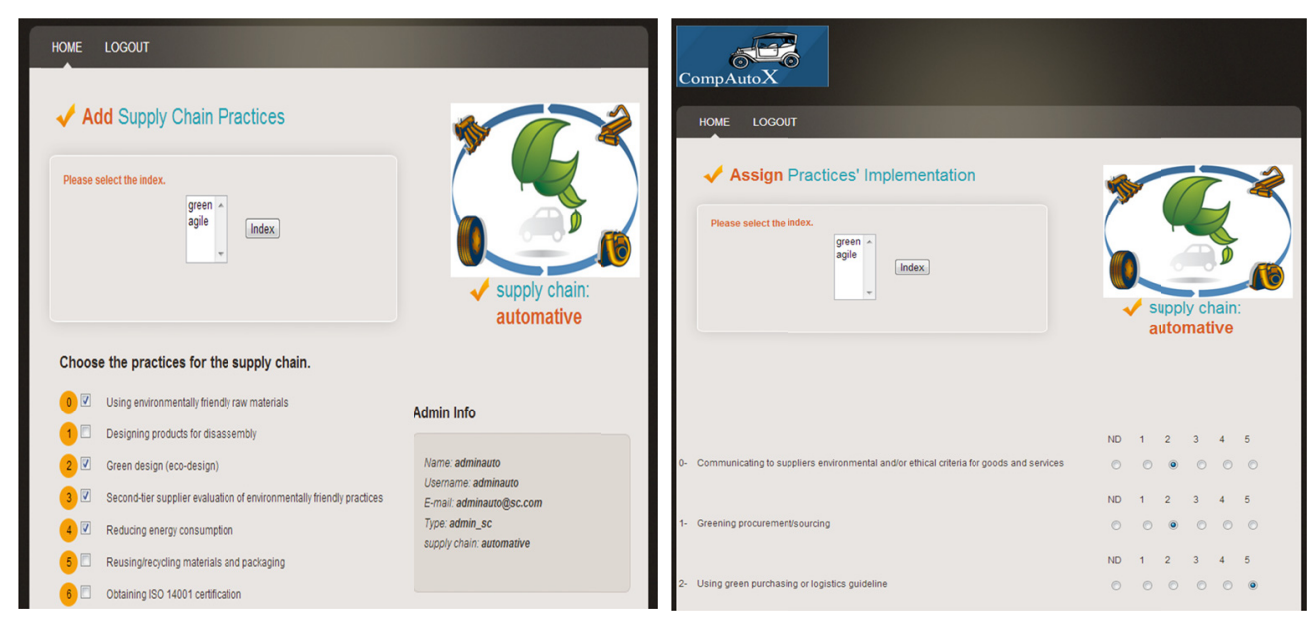

Figura 4 - Adicionar práticas para o índice verde da SC automóvel
Figura 5 - A empresa ComAutoX atribui níveis para a implementação das práticas

\subsection{A Infraestrutura de Cloud Amazon EC2}

A Amazon EC2 fornece templates denominados Amazon Machine Images (AMIs) que contêm configurações de software incluindo o sistema operativo e eventualmente outras aplicações. É possível criar diferentes tipos de instâncias a partir de uma AMI, variando o espaço de memória e a capacidade de processamento a que queremos aceder. Quando é criada uma instância $\mathrm{EC} 2$, é fornecido um par de chaves de encriptação PEM (Privacy Enhanced Mail) que tem de ser usado para executar as ligações com essa instância. Para publicar a aplicação na cloud usamos um sistema Windows acedendo por SSH através da ferramenta PuTTY. Foi por isso necessário converter as chaves PEM para o formato PPK (PuTTY Private Key). A aplicação foi testada usando uma micro instância de acesso gratuito, com $613 \mathrm{MB}$ de memória e 2 ECUs. Uma ECU (EC2 Compute Unit) tem aproximadamente a capacidade de processamento de um CPU a 1.0-1.2 GHz.

\subsection{Segurança}

A encriptação da password dos utilizadores de forma a proteger as credenciais de acesso de ataques Brute Force e Rainbow Tables (Selvakumar \& Ganadhas, 2009) e a proteção contra ataques de SQL Injection (Tajpour, A. \& Shooshtari, 2010) foram doïs dos aspetos de segurança devidamente acautelados neste protótipo. Na versão final da aplicação a segurança na comunicação entre o cliente e o servidor irá ser garantida através da utilização do protocolo HTTPS (Rescorla, 2000).

Na proteção das passwords, para cada utilizador que se regista é guardada uma variável aleatória comumente designada por salt. A password após ser concatenada 
com a variável salt é encriptada com uma função de hash ${ }^{2}$, sha256. Após repetir o processo 216 vezes o valor resultante é guardado na base de dados. Quando é feito um login, o valor do salt é lido da base de dados e com a password que o utilizador introduz é repetido o processo de encriptação. O valor gerado é comparado com o que foi guardado no processo de registo.

A proteção contra ataques de $S Q L$ injection, isto é, da possibilidade de, através da manipulação do input dos comandos enviados para a aplicação, serem enviados à base de dados queries diferentes dos previstos, foi feita através das prepared statements ${ }^{3}$ do PHP PDO [23]. Aqui as instruções SQL são construídas a partir de templates prédefinidos e em vez de se colocarem diretamente os nomes das variáveis dentro da string do query, estas são passadas como parâmetros, definidos através de placeholders, que serão substituídos quando o query for pré-processado. Assim não há variáveis passadas como strings que possam ser substituídas por queries maliciosos.

\section{Conclusão}

Tendo em conta os novos paradigmas de gestão da SC e a atual dinâmica empresarial, é importante que os decisores das cadeias de abastecimento estejam cientes (em tempo útil) do nível de implementação das principais práticas associadas a cada paradigma. Neste trabalho é proposta uma metodologia que permite a avaliação do desempenho de uma cadeia de abastecimento, e dos seus elos constituintes, num contexto global. Os índices propostos são genéricos e a sua adaptação a diferentes cadeias de abastecimento é imediata. A cloud surge aqui como suporte facilitador para o modelo colaborativo de avaliação proposto.

A computação em cloud introduz um novo modelo de negócio, onde os consumidores podem ter acesso a hardware e software através da Internet, pagando de acordo com os recursos usados, como fazemos com os serviços públicos. Da perspetiva da SC a computação em cloud transformou o modo como as redes empresariais globais interagem, proporcionando um modelo flexível e colaborativo. O estabelecimento de um ambiente dedicado de negócios virtual em uma infraestrutura comum oferece uma interface controlada - simplificando não só a distribuição para toda a SC do modelo de avaliação proposto, mas também a sua validação e posterior análise de dados históricos.

Neste sentido o protótipo de aplicação desenvolvido demonstra a utilidade do conceito e prepara o caminho para colocar em produção o sistema de avaliação. O modelo de dados foi desenhado com o intuito de acomodar não apenas as operações de aferição das práticas e cálculo dos índices associados às empresas e à SC como um todo, mas também um conjunto de funcionalidades complementares que serão desenvolvidas para o sistema em produção. Dentre estas, realçamos as seguintes tarefas: (i) identificação de práticas e respetivos pesos através de um sistema de votação disponível para os peritos; (ii) ajuste cooperativo da política de avaliação da SC por

\footnotetext{
${ }^{2}$ Secure Hash Standard (SHS) FIPS PUB 180-4, (2012).

http://csrc.nist.gov/publications/fips/fips180-4/fips-180-4.pdf

3 Prepared Statements, http://php.net/manual/en/pdo.prepared-statements.php
} 
parte dos diversos atores, permitindo a inclusão rápida e flexível de novos conhecimentos e competências; (iii) análise do histórico das avaliações permitindo o conhecimento em tempo real da evolução do comportamento da cadeia e também suportar o processo de tomada de decisão no que concerne à escolha dos próprios elementos da SC. O sistema de produção, assim melhorado, fará uso de tecnologias mais recentes que as empregues no protótipo, e.g. HTML5 e CSS3, permitindo o acesso à plataforma a partir de dispositivos móveis.

\section{Agradecimento}

Este trabalho foi parcialmente financiado pela Fundação para a Ciência e Tecnologia (FCT), projeto PEst-OE/EEI/LAooo8/2013.

\section{Referências}

Agarwal, A., Shankar, R. \& Tiwari, M. (2007). Modeling agility of supply chain. Industrial Marketing Management, 36(4), 443-457.

Angulo, P., \& Martín J. (2009). Aplicación de Sistemas Inteligentes en la Creación de Organizaciones Virtuales Dinámicas para la Prevencion del Maltrato Infantil. RISTI - Revista Ibérica de Sistemas e Tecnologias de Informação, nº 4, 69-83.

Armbrust, M., Fox, A., Griffith, R., Joseph, A., D., Katz, R., Konwinski, A., Lee, G., Patterson, D., Rabkin, A., Stoica, I. \& Zaharia, M. (2010). A view of cloud computing. Communications of the ACM, 53 (4), 50-58.

Azevedo S. G., Machado V. H. , Barroso A. P. \& Cruz Machado V. (2008). Supply Chain Vulnerability: Environment Changes and Dependencies. International Journal of Logistics and Transport, 2, 41 - 45.

Azevedo, S., Carvalho, H. \& Cruz-Machado, V. (2012). Proposal of a conceptual model to analyse the influence of LARG practices on manufacturing supply chain performance. Journal of Modern Accounting \& Auditing, 8 (2), 174-184.

Azevedo, S. Prata, P., Fazendeiro, P. \& Cruz-Machado, V. (2012). Assessment of supply chain agility in a cloud computing-based framework. Scalable Computing: Practice and Experience.

Christopher, M. (2008). The Agile Supply Chain: Competing in Volatile Markets. Industrial Marketing Management, Elsevier, 29(1), 37-44.

Christopher, M. (2005). Logistics and Supply Chain Management: Creating ValueAdding Networks. London: Prentice Hall: Financial Times.Christopher, M. \& Peck, H. (2004). Building the resilient supply chain. International Journal of Logistics Management, 15(2), 1-13.

Cumbo, D., Kline. D., \& Bumgardner, M. M. (2006). Benchmarking performance measurement and lean manufacturing in the rough mill. Forest Products Journal, 56(6), 25-30. 
Environmental Protection Agency [EPA]. (2000). The lean and green supply chain: A practical guide for materials managers and supply chain managers to reduce costs and improve environmental performance. Retrieved October 8, 2009 from http://www.epa.gov/oppt/library/pubs/archive/acct-archive/pubs/lean.pdf.

Espadinha-Cruz, P., Grilo, A. Puga-Leal, R. \& Cruz-Machado, V. (2011). A model for evaluating Lean, Agile, Resilient and Green practices interoperability in supply chains. IEEE International Conference on Industrial Engineering and Engineering Management (IEEM), 1-5.

Kefer, G. (2012). Three Ways Cloud Computing Can Improve Supply Chain Operations for the Chemical Industry. IHS Chemical Week, February 2012.

Holt, D., \& Ghobadian, A. (2009). An empirical study of green supply chain management practices amongst UK manufacturers. Journal of Manufacturing Technology Management, 20(7), 933-956.

Lambert, D. M., Cooper, M. C. \& Pagh, J. D. (1998). Supply Chain Management: Implementation Issues and Research Opportunities. The International Journal of Logistics Management, 9(2), 1-20.

Likert, R. (1932). A Technique for the Measurement of Attitudes. Archives of Psychology, Vol. 140, 1-55.

Linstone, H. \& Turoff, M. (eds) (1975). The Delphi Method: Techniques and applications. Addison-Wesley.

Mell, P. \& Grance, T. (2011). The NIST Definition of Cloud Computing. NIST - National Institute of Standards and Technology, September 2011, 7 pages.

Mentzer, J. T., Keebler, J. S., Nix, N. W., Smith, C. D. \& Zacharia, Z. G. (2001). Defining supply chain management. Journal of Business Logistics, 22(2), 1-25.

Naylor, B., Naim, M., \& Berry, D. (1999). Leagility: Integrating the lean and agile manufacturing paradigms in the total supply chain. International Journal of Production Economics, 62(10), 107-118.

Ohno, T. (1988). Toyota Production System. International Journal of Operations, Vol. 4, Productivity Press, 3-11.

Peck, H. (2005). Drivers of supply chain vulnerability: an integrated framework. International Journal of Physical Distribution \& Logistics Management, Vol. 35, 210-232.

Rao, P. \& Holt, D. (2005). Do green supply chains lead to competitiveness and economic performance? International Journal of Operations \& Production Management, 25(9), 898-916.

Rescorla, E. (2000). HTTP over TLS. RFC 2818.

Selvakumar, A. A. L. \& Ganadhas, C. S. (2009). The Evaluation Report of SHA-256 Crypt Analysis Hash Function. International Conference on Communication Software and Networks, ICCSN 'o9, 588 - 592. 
Serra, N., Gonçalves, A., Serra, J., \& Sousa P. (2008). Planeamento Estratégico no contexto de Arquitectura Empresarial. RISTI - Revista Ibérica de Sistemas e Tecnologias de Informação, $\mathrm{n}^{0} 2,1-14$.

Shuwang, W., Zhang L., Zhifeng, L., Guangfu, L., \& Zhang, H. C. (2005). Study on the Performance Assessment of Green Supply Chain. IEEE International Conference on Systems, Man and Cybernetics 2005, 942 - 947.

Srivastava, S. K. (2007). Green supply-chain management: A state-of-the-art literature review. International Journal of Management Reviews, Wiley Online Library, 9(1), 53-80.

Stevens, G. (1989). Integrating the supply chain. International Journal of Purchasing, Distribution and Materials Management, 19(8), 3-8.

Tajpour, A. \& Shooshtari, M. J. (2010). Evaluation of SQL Injection Detection and Prevention Techniques. $2^{\text {nd }}$ Int'l Conference on Computational Intelligence, Communication Systems and Networks (CICSyN), 216-221.

Womack, J. P., Jones, D. T. \& Roos, D. (1991). The Machine That Changed the World: The Story of Lean Production. Harper Perennial, 323 pages.

Xavier, L., Oliveira, M. \& Teixeira E. (2012). Teorias utilizadas nas investigações sobre gestão do conhecimento. RISTI - Revista Ibérica de Sistemas e Tecnologias de Informação, $\mathrm{n}^{0} 10,1-17$.

Zhu, Q., Sarkis, J., \& Geng, Y. (2005). Green supply chain management in China: Pressures, practices and performance. International Journal of Operations and Production Management, 25, 449-468.

Zhu Q., Sarkis J. \& Lai K. (2008). Confirmation of a measurement model for green supply chain management practices implementation. International Journal of Production Economics, Vol. 111, 261-273. 